\title{
Collaborative Scientific Authoring at CERN
}

\section{A user-centered approach}

\author{
Nikos Kasioumis ${ }^{1, *}$ and Valeria Brancolini ${ }^{1}$ \\ ${ }^{1} \mathrm{CERN}$
}

\begin{abstract}
For over a year we ran a CERN-wide trial of collaborative authoring platforms, understanding the authoring habits of the CERN community and gathering user requirements. As a result, the Overleaf cloud platform is now fully available to the CERN Community. We first explain our user-centered approach, how it led us to a decision and why it was important to follow this approach. We then highlight the usage growth of Overleaf at CERN since its adoption and we finally look into the future of CERN Authoring.
\end{abstract}

\section{Introduction}

In January of 1955, just months after the establishment of CERN, the first ever CERN Report was published, starting a publishing tradition that continues to this day. More than 1000 CERN Reports, often called CERN Yellow Reports, have since been published, including school proceedings, monographs and conference proceedings [1]. Up until some years ago, the Desktop Publishing service - and since 2011 the new E-Publishing service or simply CERN Publishing service - has been responsible for facilitating the production of these reports, providing supporting resources and performing or organising the proof-reading, copyediting, printing, shipping etc.

Stepping into the modern digital era of publishing, the CERN Publishing service welcomed automation on all possible fronts and faced the resulting technical challenges. Through our experience and interaction with authors and editors, we identified gaps in the field of authoring when it comes to consolidating formats, using tools and collaborating efficiently. With a user-centered approach in mind, we decided to engage directly with the users and understand how we can help address those gaps. In this poster we present the steps we took in that direction and where they have brought us today: from evaluating authoring platforms to organising a CERN-wide trial to offering the Overleaf cloud platform to the CERN community.

\section{Trial}

At the core of our user-centered approach lay the CERN-wide trial on collaborative authoring platforms. The specifications and scale of this trial were not obvious from the beginning. Starting in early 2015, our first steps involved sending simple surveys to users we had contact with, speaking directly to authors about the software tools they were already using and

\footnotetext{
*e-mail: nikos.kasioumis@cern.ch
} 
deploying a demo platform for a dozen users [2]. The feedback we received for these efforts was very positive but no conclusions could be drawn; that is what prompted us to formalise our efforts.

\subsection{Preparation}

Before the trial could begin, we had to define our use case. Starting with an almost blank canvas, we were ambitious about who could benefit from our efforts and how. Anyone who authors documents could be a potential user, essentially the entire CERN Community: users from different departments, with different needs and different levels of experience. We set out to find out what tools are currently used, what the user needs are and what other solutions are available.

With that in mind we designed a comprehensive trial aiming at finding out what really works for the CERN Community. Through our professional contacts, colleagues and hierarchy, we managed to get 45 willing participants, from 9 different departments at CERN (figure 1). We tried to involve as many departments as possible in order to satisfy our use case requirement of a diverse user base.
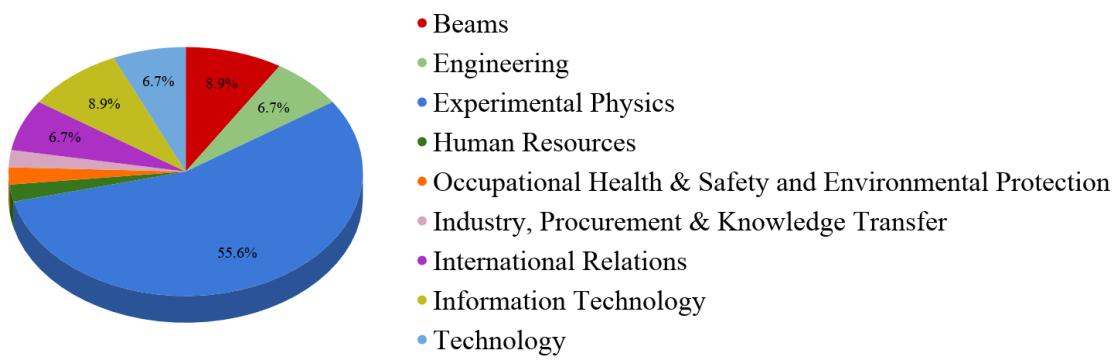

Figure 1. Distribution of the trial participants by department at CERN.

We identified 3 novel platforms: Authorea [3] (figure 2), doDOC [4] (figure 3) and Overleaf [5] (figure 4), all focusing on collaborative authoring but following distinct approaches. We negotiated free-of-charge full-access trials with their public cloud services, and the trial participants were called to try them out over the course of a few months.

\section{Aulthorea}

Figure 2. Authorea's distinctive feature is their so called "format-neutral writing environment" where multiple markup languages are supported in a single document: LaTex, Markdown, and regular rich-text.

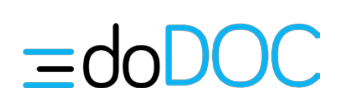

Figure 3. doDOC believes that "the paradigm of document creation and management has not changed in over 30 years" and "it's about time it changes". It focuses on "bringing companies to a new era of document creation where processes are automated, optimized and content-focused.".

Figure 4. Overleaf identifies itself as "an online LaTeX and Rich Text collaborative writing and publishing tool that makes the whole process of writing, editing and publishing scientific documents much quicker and easier.". 


\subsection{Execution}

In early 2016 we officially launched our CERN-wide trial of collaborative authoring platforms. Before the 45 participants could start trying the platforms out, we interviewed them personally to get to know them, understand their requirements, their habits and their expectations. They were then given access to the 3 platforms and throughout the trial we continuously followed up with their progress, provided them with test scenarios and motivated them.

At the end of the trial we asked all participants to fill out a detailed evaluation questionnaire in order to document their experience and their preferences on authoring generally and on each platform specifically. We received 39 responses.

After the end of the trial and the evaluation we performed 11 follow-up interviews with the most active trial participants. Through these follow-up interviews we aimed at gathering more detailed feedback, opening a discussion with the interviewees, understanding their needs in a deeper level and comprehending and evaluating the authoring features that they really need.

\subsection{Results}

The comprehensive and long process we followed made us very confident about the trial results we put together in mid 2017. Throughout the trial, the evaluation questionnaire and the follow-up interviews, the statement that had the most consensus was "I believe that a CERN authoring platform would be useful" (figure 5). The trial participants felt that the CERN community would greatly benefit from a scientific collaborative authoring platform.

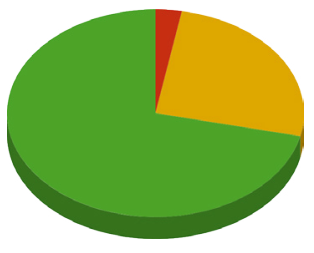

Figure 5. $71 \%$ of the trial participants fully agree that "A CERN Authoring Platform would be useful", 26\% somewhat agree while only $3 \%$ somewhat disagree.

However, one aspect that shifted was our initial focus. At the beginning, by being inclusive of the entire CERN community, we attempted to accommodate as many authoring use cases as we could identify, with possibly one tool. But our most active and eager users, the ones that produced the highest volume of documents and had the best defined requirements and expectations, turned out to be physicists and engineers in the CERN experiments. By far, the strongest use case was the scientific collaborative authoring one.

After 45 initial interviews, 39 evaluation questionnaires and 11 follow-up interviews we identified the core user requirements for scientific collaborative authoring:

- LTEX: a non-negotiable requirement in the High Energy Physics community.

- Efficiency: the platform should naturally work along existing workflows and ideally make them more efficient, automating mundane tasks.

- Collaboration: authors want to work together seamlessly and save time when collaborating.

- Versioning: integration with version control systems, such as Git, is key for the versioning and history of projects, offline access and for future preservation.

Overleaf was the preferred platform among our users (figure 6). Overleaf satisfies all the core user requirements for the scientific collaborative authoring use case: (i) it is based on pure LaTeX; (ii) it is easy to use and provides automation wherever possible; (iii) it works naturally along existing workflows / while making them more efficient; (iv) it promotes easy collaboration; (v) it integrates natively with version control systems, such as Git. 


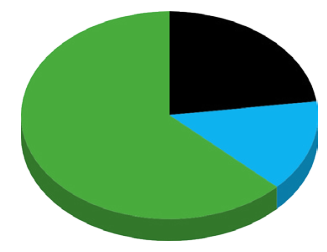

Figure 6. $63 \%$ of the trial participants chose Overleaf, 23\% chose Authorea and $14 \%$ chose doDOC as their preferred platform.

\section{Actions}

In late 2017, based on the trial results, the public cloud service of the Overleaf platform was made fully available to the CERN Community [6]. At the same time, in the scope of a more general framework, the CERN Authoring service [7] was established to offer modern authoring tools to CERN members that focus on collaboration, ease of use and integration and cover the needs of the High Energy Physics community - Overleaf being the first one of those tools.

\section{Usage}

Since it was made fully available to the CERN Community, the usage of Overleaf at CERN has been steadily increasing both in the number of users (figure 7) and the number of projects (figure 8).

\section{$2 \mathrm{~K}$}

$1 \mathrm{~K}$

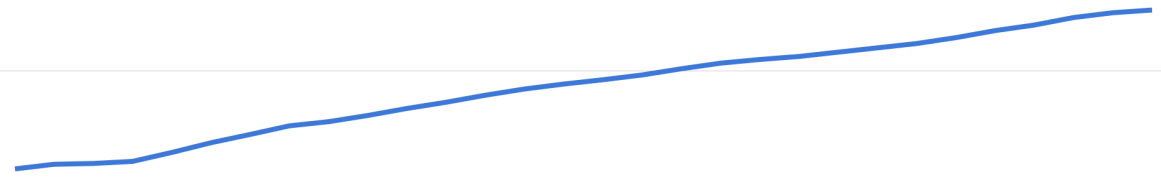

OK

Figure 7. Number of CERN users on Overleaf from December 2017 to July 2018.

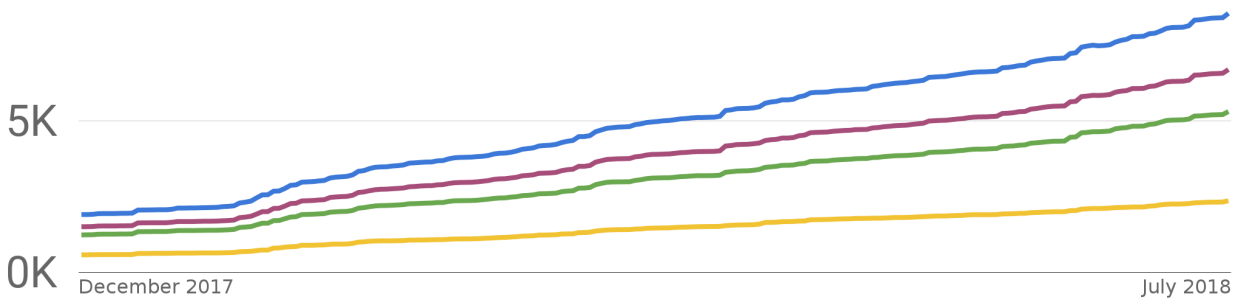

Figure 8. Number of CERN projects on Overleaf from December 2017 to July 2018 (Edits: 1+, 10+, $100+, 1000+$ ). 


\section{Future}

Staying committed to our user-centered approach, we have a created a community forum [8] through which users users can engage with us and with each other and help shape the future of authoring at CERN.

Overleaf has recently joined forces with ShareLaTeX and together they are working on Overleaf v2 which will bring together the best of both worlds [9], going public in late 2018.

\section{References}

[1] CERN Yellow Reports http://cds.cern.ch/collection/CERN\%20Yellow\%20Reports

[2] ShareLaTeX https://github.com/sharelatex/sharelatex

[3] Authorea https://www.authorea.com/

[4] doDOC https://academia.dodoc.com/

[5] Overleaf https://www.overleaf.com

[6] CERN on Overleaf https://www.overleaf.com/org/cern

[7] CERN Authoring https://cern.ch/authoring

[8] CERN Authoring community forum https://discourse.web.cern.ch/c/authoring

[9] ShareLaTeX is joining Overleaf https://www.overleaf.com/blog/518 